\title{
Flooded Streets - A Crowdsourced Sensing System for Disaster Response: A Case Study
}

\author{
Nitin Naik \\ Defence School of Communications and Information Systems \\ Ministry of Defence, United Kingdom \\ Email: nitin.naik100@mod.uk
}

\begin{abstract}
This study is about the disastrous flooding of an Indian metropolitan area of Chennai when the rain had nearly broken the record of 100-years with $374 \mathrm{~mm}$ rain falling on December 1, 2015, virtually breaking the November monthly average of $407.4 \mathrm{~mm}$ in a day. This city with a population of approximately 6.7 million people came to a standstill. Astonishingly, one of the biggest software development hubs in India was struggling for data and tools to identify which parts of the city were most affected and vulnerable to such climate phenomena. A group of software engineers quickly came up with an idea of using a Flood Map tool managed through crowdsourcing to help the citizens of Chennai and prevent further casualties. They developed a mapbased tool called Flooded Streets to report flooded streets using OpenStreetMap (OSM) data. Using this Flood Map tool, anyone in the crowd could click on a street if they knew it was flooded and update the map information. Within the following 24 hours, over 2,500 streets had been reported as flooded by the citizens of Chennai using the Flood Map tool. An ordinary citizen could zoom into a locality, visualize which streets are reported as flooded and decide their next course of action. This map was also a great aid tool for relief and aid workers to track the flooded paths and provide appropriate aids in that area. The map consists of a base layer of low-lying areas created using elevation models from ISRO and NASA, and flooded areas from UNITAR. The map interactivity was built using Mapbox GL and hosted on GitHub. This crowdsourced sensing system is an extraordinary example of disaster response using the crowdsourcing concept, which potentially helped millions of people with the minimum time and resources but with great crowd contributions from both experts and non-experts.
\end{abstract}

Keywords-Crowdsourced Sensing System; crowdsensing system; Flooded Streets; Chennai; India, OpenStreetMap, Crowdsourcing, Disaster Response

\section{INTRODUCTION}

Crowdsourcing word is a combination of the words crowd and outsourcing. This means to outsource the work to a crowd of workers. It is the practice of engaging a crowd or group for a common goal such as innovation, problemsolving, cost-effectiveness, efficiency or disaster response [2]. This crowdsourcing term was reinvented by Jeff Howe and Mark Robinson in 2006 [1]. Crowdsourcing can be classified in several ways depending on the nature and involvement of the crowd, motivation and incentives, tools and techniques [4]. Natural disasters are causing increased challenges to public health, welfare, and safety and making us vigilant for the swift response. Crowdsourcing can provide real-time data enabling quick responses during earthquakes, hurricanes, flooding, or other natural disasters [3]. The Internet and social media are one of the most crucial aids for disaster response in coordinating rescue operation, sheltering and supplying food for the distressed. Crowdsourced sensing/crowdsensing systems enable us to gain insight into our local environments or to measure phenomena of common interest by leveraging voluntarily contributed data by using the various types of sensors equipped with suitable sensing software. Flooded Streets is the most recent example of crowdsourced sensing system developed and deployed for the disaster response in the disastrous flooding of Chennai. This case study explores about the crowdsourced sensing system Flooded Streets and lesson learnt for the future.

The remainder of this paper is organised as follows: Section II explains about crowdsourced sensing/crowdsensing (CS) and crowdsourced sensing/crowdsensing system (CSS); Section III presents the classification of crowdsourced sensing/crowdsensing (CS); Section IV elicits the importance and success of crowdsourced sensing/crowdsensing system "Flooded Streets" for the humanitarian use during the flooding incident in Chennai, India. Section V concludes the paper.

\section{CROWdSOURCED SENSING/ CROWDSENSING SYSTEMS (CSS)}

Nowadays most of the smartphone has inbuilt sensors such as GPS, audio, image, accelerometer, magnetometer, gyroscope, light, proximity (LED and IR), barometer and pedometer [5]. The universality of smartphones has provided an opportunity to create low-cost sensing systems for activities that may have a huge societal impact [6], [7], [8], [9], [10], [11]. Crowdsourced sensing/crowdsensing (CS) is a large-scale data sensing activity by employing the crowd; therefore, sensing data can be collected swiftly and economically. Crowdsourced sensing/crowdsensing systems (CSSs) enable us to gain insight into our local environments or to measure phenomena of common interest by leveraging voluntarily contributed data by using the various types of sensors (e.g., smartphone sensors and IoT devices) equipped with suitable sensing software. Depending on the method of the data collection and accuracy of sensors, data need to be further processed and analysed for extracting the relevant information. This is managed by serverside platform, which receives, processes, analyses and stores the measured phenomena [6]. Security and privacy are another important aspects of crowdsourced sensing system where the crowd can trust in the involved system, and the system can prevent their privacy [4]. In a nutshell, crowdsourced 
sensing/crowdsensing systems are one of the best and swift mechanism to cope with natural disasters.

\section{Classification of Crowdsourced Sensing/ CROWDSENSING (CS)}

Crowdsourced sensing/crowdsensing (CS) can be classified in several different ways such as the involvement of the crowd or the type of the measured phenomenon in the crowdsensing process [4], [6], [7], [8], [9]. The crowd participation in CS can also be classified as personal and community [7], [8]. In personal CS, phenomena related to an individual crowd are measured (e.g., shopping, eating, running, walking, exercising, commuting). In community CS, phenomena related to a certain group or a large crowd are measured (e.g., traffic, pollution, transportation). Community CS can also be further subclassified as participatory CS and opportunistic CS [4], [7]. In participatory $\mathrm{CS}$, the crowd is actively involved and send sensor data to a server (e.g., taking a picture, reporting a road closure, traffic, flooding). In opportunistic CS, the crowd is minimally involved and sensor data is automatically sent to a server (e.g., continuous measuring/sampling of temperature, pressure, location without the explicit action from the user). The measured phenomenon in CS can also be classified as environmental, infrastructure, and social [4], [7]. Environmental CS is used for measuring phenomena related to the natural environment (e.g., level of water, air pollution, wildfire habitats). Infrastructure CS is used for measuring phenomena related to the public infrastructure (e.g., traffic congestion and road conditions). The social CS is used for measuring phenomena related to the social life of individuals/groups (e.g., shopping, eating, exercising, holidaying and recreational habits) [4].

\section{Flooded Streets - A Case Study}

This section presents a case study about the successful employment of the crowdsourced sensing/crowdsensing system "Flooded Streets" (see Fig. 1) for disaster response to the most recent flooding incident in the Indian city of Chennai. It highlights the importance and success of the crowdsourced sensing/crowdsensing system "Flooded Streets", which potentially helped millions of people with the minimum time and resources during the Chennai flood incident in December 2015 [12], [13], [14]. Flooded Streets was a participatory and environmental crowdsensing project.

The disastrous flooding, due to the heaviest rainfall in more than 100-years, ravaged an Indian metropolitan area of Chennai in December 2015. The average rainfall of Chennai was approximately $407.4 \mathrm{~mm}$ in November month; however, this year it was $1218.6 \mathrm{~mm}$, approximately, three times higher than its monthly rainfall [14], [15]. Additionally, December 1 , was a tragic day when the rainfall was $374 \mathrm{~mm}$ in a single day, virtually equal to the monthly average rainfall of November. This city with a population of approximately 6.7 million people came to a standstill. Astonishingly, one of the biggest software development hubs in India had virtually no data or tool available to identify which parts of the city were most affected and vulnerable to such climate phenomena.

A group of software engineers Arun Ganesh, Aruna Sankaranarayana and Sanjay Bhangar quickly came up with an

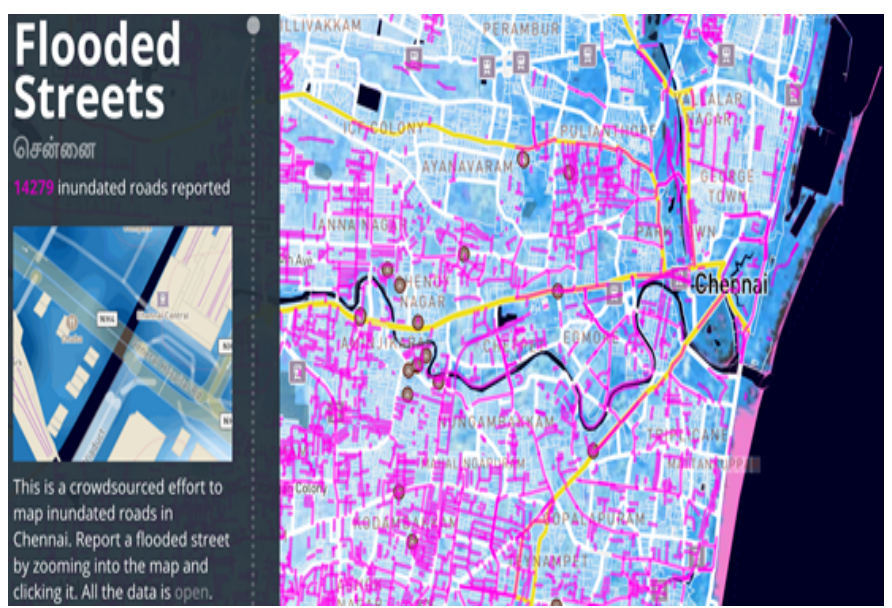

Fig. 1: Flooded Streets Map [13]

idea of using a Flood Map tool managed through crowdsourcing to help the citizens of Chennai and prevent further casualties [12]. They developed a map-based tool called "Flooded Streets" to report flooded streets using OpenStreetMap (OSM) data. OSM is an open-source project to create a free editable map of the world [16]. Volunteers gather location data using GPS, local knowledge, and other free sources of information and upload it [17]. OSM can be used by anyone under an open license. Using the "Flooded Streets" tool, anyone in the crowd could click on a street if they knew it was flooded and update the map information. Within the following 24 hours, over 2,500 streets had been reported as flooded by the citizens of Chennai using the "Flooded Streets" tool. An ordinary citizen could zoom into a locality, visualize which streets are reported as flooded and decide their next course of action. If their street, building or neighbourhood name was missing on the map, then they could add it to the basemap directly by editing OpenStreetMap [18]. Subsequently, the flood map would be updated with the new street in 10 minutes. The flooded streets layer was contributed by the crowd. All the crowd contributors were anonymous with no version control for the sake of simplicity of the map. This Flood Map tool was also a great aid for relief and aid workers to track the flooded paths and provide appropriate aids in that area. The map consists of a base layer of low-lying areas created using elevation models from ISRO and NASA, and flooded areas from UNITAR [12]. The elevation models are created using thousands of images acquired with a large range of viewing geometries and illumination conditions. The model can be used to show a variety of topographic features including the highest and lowest elevations. The Flood Map interactivity was built using Mapbox GL and hosted on GitHub.

Natural disasters and their severity are not always predictable with the precision. However, one thing is certain, i.e., the power of the crowd and if people are volunteered in making systems for disaster response, then that can potentially help community, aid-workers and government organisations to minimise the casualties and other losses. Thus, this crowdsourced sensing system "Flooded Streets" is an extraordinary example of disaster response using the crowdsourcing concept, which potentially helped millions of people with the minimum 
time and resources but with great crowd contributions from both experts and non-experts. The map developers have now offered help and support to the community for expanding this "Flooded Streets" map for other cities which are most prone to flooding. The Flood Map data is available to all for further use and analysis purpose. This is an exemplary model which can also be adapted for helping the community in case of other natural disasters.

\section{CONCLUSION}

This paper presented a case study about the successful employment of the crowdsourced sensing/crowdsensing system "Flooded Streets" for disaster response to the most recent flooding incident in the Indian city of Chennai in December 2015. This Flood Map tool was based on OpenStreetMap data, and its interactivity was built using Mapbox GL and hosted on GitHub. It was used to view and report flooded streets by the crowd. It was also used by the aid workers to track the flooded paths and provide appropriate aids in that area. This Flood Map tool potentially helped millions of people with the minimum time and resources but with great crowd contributions from both experts and non-experts. Therefore, this crowdsourced sensing system "Flooded Streets" is an exemplary model for disaster response using the crowdsourcing concept; which can also be adapted for helping the community in case of other natural disasters.

\section{REFERENCES}

[1] J. Howe. (2006, June 2) Crowdsourcing: A Definition. [Online]. Available: http://crowdsourcing.typepad.com/cs/2006/06/ crowdsourcing_a.html

[2] N. Naik, "Crowdsourcing, Open-sourcing, Outsourcing and Insourcing Software Development: A Comparative Analysis," in 10th IEEE Symposium on Service-Oriented System Engineering (IEEE SOSE). IEEE, 2016, pp. 380-385.

[3] T. F. Tierney. (2014, July 9) Crowdsourcing disaster response: Mobilizing social media for urban resilience. [Online]. Available: http://www.europeanbusinessreview.com/4911/

[4] D. Dimov, "Crowdsensing: state of the art and privacy aspects," InfoSec Institute, vol. 29, 2014. [Online]. Available: http:// resources.infosecinstitute.com/crowdsensing-state-art-privacy-aspects/

[5] J. G. Rodrigues, A. Aguiar, and J. Barros, "Sensemycity: Crowdsourcing an urban sensor," arXiv preprint arXiv:1412.2070, 2014.

[6] E. Ozer, M. Q. Feng, and D. Feng, "Citizen sensors for shm: towards a crowdsourcing platform," Sensors, vol. 15, no. 6, pp. 14 591-14614, 2015.

[7] R. K. Ganti, F. Ye, and H. Lei, "Mobile crowdsensing: current state and future challenges." IEEE Communications Magazine, vol. 49, no. 11, pp. 32-39, 2011.

[8] T. Monahan and J. T. Mokos, "Crowdsourcing urban surveillance: The development of homeland security markets for environmental sensor networks," Geoforum, vol. 49, pp. 279-288, 2013.

[9] L. Xu, X. Hao, N. D. Lane, X. Liu, and T. Moscibroda, "More with less: lowering user burden in mobile crowdsourcing through compressive sensing," in Proceedings of the 2015 ACM International Joint Conference on Pervasive and Ubiquitous Computing. ACM, 2015, pp. 659-670.

[10] S. Hu, H. Liu, L. Su, H. Wang, and T. Abdelzaher, "SmartRoad: A mobile phone based crowd-sourced road sensing system," University of Illinois at Urbana-Champaign, Tech. Rep., 08 2013. [Online]. Available: https://www.ideals.illinois.edu/handle/2142/45699

[11] D. Yang, G. Xue, X. Fang, and J. Tang, "Crowdsourcing to smartphones: incentive mechanism design for mobile phone sensing," in Proceedings of the 18th annual international conference on Mobile computing and networking. ACM, 2012, pp. 173-184.
[12] A. Ganesh. (2015, December 2) Crowdsourcing flood data for Chennai [Online]. Available: https://www.mapbox.com/blog/chennai-flood-map/

[13] GitHub. (2015, December 2) Flooded Streets. [Online]. Available: https://osm-in.github.io/flood-map/chennai.html\#12/13.0597/80.2624

[14] A. Pereira. (2015, December 4) Chennai floods: Decoding the citys worst rains in 100 years. [Online]. Available: http://indianexpress.com/article/india/india-news-india/chennaifloods-rains-jayalalithaa-imd-reasons-rescue-news-updates/

[15] GeospatialMedia. (2015, December 4) Citizens build map data flooded Chennai using open source maps. [Online]. Available: https://www.youtube.com/watch?v=iSroARsgTyM

[16] Firstpost.com. (2015, December 2) Now here's a map to help you report and avoid flooded streets in chennai. [Online]. Available: http://www.firstpost.com/india/now-heres-a-map-to-helpyou-report-and-avoid-the-flooded-streets-in-chennai-2530456.html

[17] ESRI. (2014, December 11) OpenStreetMap. [Online]. Available: https://www.arcgis.com/home/item.html?id= b834a68d7a484c5fb473d4ba90d35e71

[18] GitHub. (2015, December 2) Flood map. [Online]. Available: https://github.com/osm-in/flood-map 